\title{
Identificación de nuevas voces de la medicina dieciochesca mediante procesos de reformulación ${ }^{*}$

\author{
Identification of new medical terms of the eighteenth century
} through reformulation processes
}

\begin{abstract}
JOSEFA GÓMEZ DE ENTERRÍA
Universidad de Alcalá

j.gomezdeenterria@uah.es

Resumen: El objetivo del artículo es detectar la presencia de algunos neologismos médicos a partir del seguimiento de los mecanismos de reformulación presentes en el discurso especializado de la medicina dieciochesca. Así, hemos observado que son los procesos de la reformulación los que proporcionan el acceso a la dimensión léxica de los textos, favoreciendo la identificación de las nuevas voces. Para ello hemos reunido una base documental constituida por un corpus textual de obras médicas con traducciones y obras originales, y otro corpus lexicográfico con un conjunto de diccionarios idóneos. A partir de dos sincronías, estrechamente relacionadas con la evolución de la medicina dieciochesca, hemos hecho el seguimiento del cambio semántico que acusan las voces arteriotomía y poro durante el período acotado. Palabras clave: lexicogénesis; reformulación; siglo XVIII; vocabulario de la medicina.

Abstract: The objective of this article has been to detect the presence of some medical neologisms from the follow-up of the reformulation mechanisms present in the specialized discourse of eighteenth-century medicine. Thus, we have observed that it is the reformulation processes that provide access to the lexical dimension of texts, favoring the identification of new voices. For this we have assembled a documentary base constituted by a textual corpus of medical works with both translations and original works, and another lexicographic corpus with a set of suitable dictionaries. From two synchronies, closely related to the evolution of eighteenth-century medicine, we have monitored the semantic change that the terms arteriotomy and pore during the above-mentioned period.

Key words: lexicogenesis; eighteenth century; reformulation; medicine lexicon.
\end{abstract}

Fecha de presentación: 13/10/2019 Fecha de aceptación: 12/11/2019.

\section{INTRODUCCIÓN}

Para la realización de este artículo partimos del aserto de que es en la reformulación donde se produce la reinterpretación y reelaboración textual. Esta se manifiesta mediante importantes procesos de producción del discurso que facilitan la observación del tratamiento discursivo. Pero también consideramos que, muchas veces, son los procesos de la reformulación los que proporcionan el acceso a la dimensión léxica de los textos, favoreciendo la identificación de los neologismos. Cuestión esta fundamental si tenemos en cuenta que la inmersión en los procesos lexicogenéticos, forjadores de la

* Este trabajo se enmarca en el Proyecto de Excelencia «Estudio de las tradiciones discursivas y su evolución en un corpus textual de obras médicas del siglo XVIII» ETMD18 (ref. FFI2015-70721-P). 
nueva terminología médica del siglo XVIII, constituye el segundo objetivo general del proyecto ETMD18.

Así, hemos tratado de detectar la presencia de algunos neologismos médicos en el corpus textual acotado, tomando como punto de partida el seguimiento de los mecanismos de reformulación de los términos dentro del discurso especializado de la medicina dieciochesca, y analizando la equivalencia conceptual de las voces a través de los marcadores reformulativos parafrásticos y no parafrásticos. Tratamos pues de analizar el proceso en el que se determinan las posibles relaciones que el discurso médico dieciochesco establece entre los términos y su reformulación, mediante una expresión más analítica y transparente que sirve para apuntalar los neologismos especializados. Partimos pues de una aproximación discursiva de base cognitiva que concibe el discurso especializado como un ente dinámico que avanza a medida que se va construyendo el discurso científico (Bach, 2005).

$\mathrm{Al}$ abordar el análisis de los textos de medicina, hemos tomado como punto de partida el principio planteado por Oesterreicher (2004: 731) acerca de que la Historia de la lengua debe considerar todos los materiales lingüísticos que los estudios tradicionales, centrados en la lengua literaria han dejado habitualmente al margen. Ya en 1991, Eberenz apuntaba la necesidad de orientar el estudio del léxico, y más especialmente el del vocabulario terminológico hacia la recogida e interpretación de los materiales lingüísticos relativos, directa o indirectamente, situándolos en el contexto sociocultural en que se localiza la lengua (1991: 96). Sin olvidar la importancia que puede llegar a alcanzar el estudio histórico de la divulgación científica cuando contribuye a aclarar los procesos o fenómenos actuales de la divulgación. Porque muchas veces estos fenómenos actuales solo pueden ser explicados a partir de los textos de la divulgación histórica (Brumme, 2001: 10). Es por esto por lo que hemos compilado un corpus textual que acoge textos de especialidad de la medicina dieciochesca, cuya finalidad comunicativa es perseguir la eficacia y el rigor de la comunicación. En dicho corpus hemos valorado los diferentes grupos de individuos que, dentro de la comunidad científica, determinan o tratan de imponer ciertos usos lingüísticos más o menos renovadores, comprobando así hasta qué punto pudieron lograr tal propósito.

\section{EL CORPUS}

El corpus textual compilado para este trabajo ${ }^{1}$ está constituido por textos médicos del siglo XVIII y acoge dos subconjuntos representativos de sendos momentos, que son decisivos para la medicina dieciochesca. El primero, conocido como la etapa novatora, va a favorecer la llegada del lenguaje de la nueva ciencia, con una periodización que abarca las últimas décadas del siglo XVII y primeras del XVIII. El segundo momento, que podemos considerar de consolidación del lenguaje médico durante el período ilustrado, se sitúa cronológicamente en la segunda mitad de la centuria, cuando la corriente divulgadora llega en tromba hasta la literatura médica.

\footnotetext{
${ }^{1}$ Este corpus se completó con los de los proyectos del Plan nacional: VCT18 (ref. FFI2008-01586) y VCT18-II (ref. FFI2011-24090), que han precedido al presente proyecto y forman una unidad con él.
} 
El corpus también nos permite observar cómo los médicos dieciochescos construyen sus escritos mediante diferentes procedimientos de organización discursiva para alcanzar la plena interconexión de los interlocutores, al tiempo que introducen en su discurso las nuevas voces. Es entonces cuando los procedimientos de reformulación se nos muestran como prácticas retóricas de razonamiento empírico, propias de la disciplina. Incluso en aquellos casos en que la situación comunicativa planteada no es equidistante, como ocurre en los textos didácticos y en los divulgativos, cuando el destinatario no posee el mismo nivel de conocimientos que el emisor.

\section{CONTEXTO HISTÓRICO}

Situamos el análisis en dos períodos históricos correspondientes con dos momentos reveladores para la evolución de la ciencia, pues ambos tuvieron capital importancia para el desarrollo de la medicina dieciochesca: el primero, en la etapa novatora; el segundo, a partir del medio siglo con la llegada de los textos divulgativos de la medicina ilustrada hasta nuestro país, gracias a las traducciones que se vierten en la segunda mitad de la centuria (Gómez de Enterría, 2013).

\subsection{La etapa novatora}

Esta etapa constituye en la medicina un período de europeización y renovación científica que abarca el reinado de Carlos II (1665-1700) y la primera parte del de Felipe V (1700-1724). Son los médicos novatores, defensores de la iatroquímica y contrarios al galenismo, los que introducen en España la nueva medicina en un momento de verdadera renovación científica. En momentos como este, de fuerte controversia científica, algunos novatores desempeñaron un papel importante como por ejemplo el médico italiano Malpighi, figura preclara del pensamiento médico europeo que ejerció de mediador científico, dando a conocer la nueva ciencia en Europa. Pero también los médicos franceses, que llegaron con el séquito de la nueva dinastía borbónica, tuvieron una actividad muy destacada, contribuyendo poderosamente a la legitimación social de la medicina moderna, y colaborando a la renovación de la ciencia junto con los médicos novatores españoles.

Ya en 1687 Juan de Cabriada, médico valenciano formado en la Universidad de Zaragoza, publica su obra Carta Phylosóphica médico chymica, en la que denuncia el atraso que padecía la ciencia médica en España. Su propuesta de renovación trataba de superar el atraso de la ciencia patria proponiendo un gran esfuerzo de comunicación con la ciencia renovadora vigente en Europa.

\subsection{La traducción de obras médicas en la época ilustrada}

Los años centrales de la centuria fueron decisivos para consolidar la llegada hasta nuestro país de un rico acervo de traducciones de la medicina ilustrada. Los médicos hispanos persiguiendo la necesaria incorporación del país al progreso, vierten los tratados fundamentales que habían marcado los avances de la ciencia médica en Europa. Al mismo tiempo que las ideas ilustradas y las nuevas prácticas mercantilistas van a favorecer el desarrollo de un próspero comercio editorial.

Muchas de las versiones que hacen los médicos en este período son de obras de carácter divulgativo, que contribuyen eficazmente al asentamiento de la medicina social 
en España. Se trata de un momento de cambio en cuanto a las condiciones sociales, políticas y culturales, con el consecuente desarrollo del concepto de higiene social e individual. Temas como la conservación de la salud y la medicina preventiva alcanzarán gran notoriedad en nuestro país gracias a la llegada de las obras de Tissot (17281797), Le Bègue de Presle (1735-1807) y Buchan (1729-1805), entre otros. Serán pues las versiones de obras divulgativas las que afiancen este nuevo fenómeno de valoración de la vida humana y de la salud, alcanzando gran aceptación en la sociedad dieciochesca y favoreciendo en ocasiones la publicación simultánea de diversas versiones de una misma obra. Uno de los casos más notorios lo constituyen las obras del médico suizo Samuel Auguste André David Tissot cuyas traducciones fueron el impulso definitivo para la proyección de este género de literatura médica en lengua castellana.

\section{REFORMULACIÓN Y NEOLOGÍA}

Los textos de los médicos dieciochistas muestran en sus escritos una profunda preocupación por la utilidad pública, al tiempo que centran su punto de mira en el establecimiento de relaciones entre el progreso científico de España y el desarrollo positivo de la ciencia médica. Cuando estos médicos redactan sus textos recurren con frecuencia al empleo de diversos procedimientos que transforman su discurso fuente o discurso primero en un enunciado diferente o discurso segundo, en el que se produce una redistribución de los elementos que lo forman. Son estos discursos segundos los que acogen las marcas de la reformulación, que aparecen ante nosotros con los rasgos caracterizadores de un momento determinado de expansión de la ciencia como el que nos ocupa — no olvidemos que el deseo por comunicar lo nuevo es prioritario tanto en la corriente novatora como en la etapa ilustrada - y que nos permite identificar las estrategias discursivas propias de cada autor. Distinguiéndose dos tipos de reformulación: la parafrástica, que establece una relación de equivalencia entre el miembro reformulador y el reformulado; y la no parafrástica, en la que el emisor reinterpreta, desde una nueva perspectiva, lo enunciado en el primer miembro (Roulet, 1987, Rossari, 1994, Fuentes, 1994 y Pons, 1998).

El análisis de estos discursos de la reformulación también nos permite observar el funcionamiento de dos facetas propias de los textos, de una parte, en el plano interlingüístico al mostrarnos la estructura de los discursos junto con los enunciados; de otra, cuando detectamos su funcionamiento en el plano extralingüístico, dada la influencia que ejercen los factores de carácter histórico y sociológico sobre los autores que redactan los textos de la medicina dieciochesca. Ya sea en la etapa final de la casa de Austria con el advenimiento de la dinastía borbónica, cuando España se encuentra inmersa en un período de fuertes contrastes e influencias cruzadas; o en el período ilustrado, verdadera situación de encrucijada para la ciencia médica. Es entonces cuando la medicina social se implanta en España respaldada en gran medida por las traducciones de obras divulgativas relacionadas con la higiene y la conservación de la salud. En consecuencia, estas dos facetas propuestas - la estructura de los discursos y el funcionamiento de los enunciados - tendrán que abordarse de forma rigurosa para llevar a cabo la explicación de los hechos lingüísticos. Solo así lograremos que la descripción históricolingüística-textual nos proporcione datos de interés para el estudio de la historia de la lengua científica española de los dos períodos acotados para este trabajo. 
Los textos del corpus nos proporcionan ejemplos con reformulación parafrástica, cuando se establece una relación de equivalencia entre el miembro reformulador y el reformulado $\mathrm{y}$, no parafrástica, cuando el emisor reinterpreta, desde una nueva perspectiva, lo enunciado en el primer miembro. La mayoría de los ejemplos que hemos tomado proceden de la primera y resultan de gran interés para nuestra investigación, pues son estos procesos de la reformulación los que nos permiten el acceso a la dimensión léxica de los textos y, con frecuencia, nos indican la presencia de voces neológicas.

\subsection{Arteriotomía}

La voz arteriotomía ofrece espacios discursivos que persiguen la equivalencia conceptual plena en los dos momentos históricos acotados, como muestran los ejemplos siguientes:

(1) M. Cómo se llama esta sangría? D. Arteriotomía, que es lo mismo que sección o incisión de arteria (Le Preux $\left.{ }^{2}, 1715: 9\right)$.

(2) Acad. Quantas especies ay de Sangria? Doct. Dos, una que dizen Phlebotomía, que es lo mismo que Incisión de Vena; y otra Arteriotomía, que es lo mismo, que Incisión de Arteria (Monrava y Roca ${ }^{3}, 1725$-I: 111).

(3) Por lo que hace al modo con lo que se ha de sangrar, se deberá siempre hacer la sangría de una vena muy ancha o de gran diámetro; porque haciendo la sangría de una vena pequeña o de una arteria no se puede evacuar la suficiente quantidad de sangre que sea alivio o auxilio al sistema de los vasos: la arteriotomía u evacuación por la arteria es por otra parte inseparable de ciertos inconvenientes (Serrano Manzano [Brown], 1800-II: 61).

La equivalencia entre el enunciado y su formulación resuelve los problemas de comprensión que plantea la palabra arteriotomía. Esta voz, a pesar de que tiene su origen en la medicina galénica se presenta en el período que nos ocupa como relativamente nueva, pues su práctica en la cirugía hispana había llegado a ser casi desconocida, debido a los inconvenientes que podía acarrear su aplicación. Así, no es extraño que los cirujanos hispanos, a pesar de conocer esta técnica, que era de uso bastante frecuente en Europa, no la empleasen. Solo esto puede explicar la escasa, casi nula, aparición de la voz arteriotomía en los textos de nuestra cirugía renacentista y barroca. Y tal vez sea esta la explicación de la práctica desaparición de la voz en los repertorios médicos y quirúrgicos ${ }^{4}$.

\footnotetext{
${ }^{2}$ Cirujano francés que se instala en España con la llegada de la dinastía borbónica. Aquí publica su Doctrina para sangradores, alcanzando sucesivas ediciones hasta mediados del siglo XIX, estas verán la luz en Madrid, Salamanca, Valencia y Zaragoza. También llegó su éxito hasta México, donde se edita con una reimpresión en 1824. Le Preux incluye la arteriotomía en el título de su manual Doctrina moderna para sangradores, en la qual se trata de la Flebotomía, y Arteriotomía [...] [Madrid, 1717], aunque a lo largo de la obra sólo nombra la arteriotomía una única vez.

${ }^{3}$ Antonio Monrová y Roca, médico catalán doctorado en medicina por la Universidad de Lérida. Fue catedrático de prima de anatomía en Lisboa, donde fundó la Academia físico-anatómica-médico-quirúrgica, poco después obtuvo la plaza de médico anatómico del rey de Portugal, Juan V. En 1725 publicó en Lisboa su obra Breve curso de nueva cirugía, redactada mediante diálogos entre el doctor presidente y los académicos; en esta obra introduce la nueva medicina y hace patente su acusado antagonismo con el Dr. Suárez de Ribera.

${ }^{4}$ A mayor abundamiento sobre esta cuestión, véase Gómez de Enterría (en prensa).
} 
En (1) y (2) observamos que los médicos del período novator emplean el marcador de reformulación parafrástica de tipo explicativo: que es lo mismo (Martín Zorraquino y Portolés, 1999), con el fin de resolver el conflicto semántico que el término especializado pudiera plantear a los lectores. En los dos ejemplos citados las nociones de contexto, registro, género y tipo de texto coinciden, así como las condiciones que les impone el empleo de un canal de comunicación idéntico (Calsamiglia, 2000: 4), porque ambos proceden de sendas obras de carácter didáctico que emplean el género dialógico para dirigirse al lector. Esta tradición secular en la historia de la medicina se desarrolla desde la baja Edad Media y alcanza su florecimiento con el humanismo renacentista. Es evidente que aquí el diálogo favorece la interacción entre los interlocutores, gracias al empleo de espacios discursivos comunes.

Sin embargo, en el ejemplo (3) tomado del período ilustrado se aprecia un cambio, pues la reformulación discursiva viene condicionada por un enunciado informativodescriptivo que constituye el primer miembro y resuelve la equivalencia con una unidad más analítica, introducida por el marcador de reformulación perifrástica $u[o]$, también explicativo.

Si nos remontamos a la primera datación que nos ofrece el corpus para la voz arteriotomía (4), fechada casi tres décadas antes del ejemplo (1), encontramos un caso de reformulación no parafrástica introducida con una cita de autoridad, que constituye el primer miembro, al tiempo que da paso a la nueva voz. Este ejemplo, además de darnos la retrodatación del término ${ }^{5}$, nos proporciona un dato que reviste interés para la historia de la palabra, pues el médico-autor manifiesta cierta reticencia a la hora de aplicar la arteriotomía:

(4) Otros autores, siguiendo a Galeno, aconsejan la arteriothoma (sic), rompiendo las arterias de las sienes; y aunque dizen, que con solo la ligadura se detiene el fluxo de sangre arterial, no entro bien en este remedio (Robledo, 1687 [1703]: 121).

El interés de este dato, que a primera vista pudiera parecer anecdótico, se revaloriza al comprobar que no es este el único caso - de entre los textos que hemos recogidoen donde se cuestiona la práctica de la arteriotomía como método curativo. Lo que nos lleva a pensar que tal vez esta podría ser la causa de la escasa o nula aparición de la voz en los tratados médicos de siglos anteriores, frente al entusiasmo que muestran por esta técnica quirúrgica muchos médicos del XVIII, hecho que por sí solo justificaría el empleo frecuente del término a lo largo del siglo ilustrado. Como muestra el Diccionario del Padre Terreros cuando acoge la voz en su diccionario aportando un dato que atestigua su escaso empleo de etapas anteriores al XVIII: «Arteriotomía, sangría de la arteria. Del lat. tomado del griego. Esta sangría sólo se hace en las sienes o detrás de las orejas por la dificultad que habría en otra parte para cerrar la arteria». La definición del jesuita es acorde con la reflexión que ofrece el ejemplo (4) cuando, en 1687, Robledo pone en duda la eficacia de este remedio curativo; un siglo más tarde, Serrano y Manzano, al traducir la obra de Brown, abunda en la misma idea cuando escribe: «[...] la

${ }^{5}$ Arteriotomía está recogida en el Diccionario de Autoridades, vol. I, 1726, con la autoridad de Le Preux tomada de la misma obra de donde procede el ejemplo (1). 
arteriotomía u evacuación por la arteria es por otra parte inseparable de ciertos inconvenientes» (Serrano Manzano [Brown], 1800-II: 61).

Massoneau ${ }^{6}$-médico francés en la corte de Felipe V— recurre a la reformulación no parafrástica, empleando una secuencia discursiva de carácter argumentativo, propia de los lenguajes de especialidad, al tiempo que la refuerza con una cita de autoridad:

(5) Pienso, que no se duda de esta verdad, porque fue practicada por los Antiguos, como por el tan nombrado Ambrosio Paré, en la persona del Príncipe de la Rochesurion, [...] como se ve en su libro de las operaciones de Cirugía: también puedo decir aver hecho yo muchas vezes, esta operación de la arteriotomía, como el único remedio que he hallado en los dolores intolerables de cabeza (Massoneau, 1722: 87-88).

El Dr. Martínez también recurre a este mismo procedimiento discursivo cuando trata de relacionar la arteriotomía con la medicina galénica, aún en boga en España cuando él escribe, y lo justifica con una cita de autoridad que toma de los textos antiguos:

(6) Por otro sueño semejantemente cuenta, que curó con la arteriotomía en la mano un dolor de lado al Sacerdote de Esculapio (a quien fue muy devoto) en Pérgamo (Martínez, 1723b [1797]-II: 22).

La voz arteriotomía, del gr. artêriotomía ó nica, como hemos visto en (6). Según DICCIOMED (Cortés Gabaudan y Ureña Bracero, 2011) ya estaba en el latín renacentista: artēriotomia. Es posible que su escaso empleo en los textos hispanos de la medicina de los siglos XVI y parte del XVII haya favorecido su escasísima presencia en los corpus y repertorios lexicográficos. Lo que sí parece evidente es su revitalización en el XVIII, cuando aparece en los textos con las marcas de la reformulación que le proporciona la novedad de su nueva actividad en el siglo ilustrado.

Es así como el término arteriotomía se instala en la lengua de la medicina del siglo XVIII con el significado: 'extracción de sangre que provoca una sangría', como leemos en (7) cuando su autor presenta este concepto mediante la reformulación parafrástica que establece la relación de equivalencia conceptual plena entre el término y un sintagma descriptivo:

(7) Muchos alaban la Arterotomía y las sanguijuelas a la parte, pero el todo consiste en la curación principal febril, y destruir dicha tensión violenta (Belmonte y Segura, 1730: 332).

Ya en el período ilustrado el nuevo término se instala definitivamente en la lengua de la cirugía y es frecuente encontrarlo formando parte de enumeraciones introducidas por el miembro del discurso que posee el significado más general:

(8) La sangría es un remedio conveniente y aun necesario quando hay frequencia o plenitud en el pulso, o algunas señales que indican que la sangre sube con

\footnotetext{
${ }^{6}$ Jean Massoneau nació en Agén, Francia. Hijo y nieto de profesores de cirugía, estudió medicina en Montpellier y Ferrara, donde ejerció, pasando posteriormente a servir como cirujano de la Armada con Luis XIV. Su obra Cirugía natural, dedicada a la reina Isabel de Farnesio, tiene gran importancia en la época novatora porque defiende abiertamente la circulación de la sangre, a pesar de la reticencia que aún perduraba entre los galenistas hispanos, pues la circulación sanguínea ya había sido descrita por William Harvey en la segunda década del XVII.
} 
más fuerza a los vasos de la cabeza. En estos casos algunos prácticos han preferido sangrías particulares como la arteriotomía, las escarificaciones en la nuca o la abertura de la yugular (Piñera y Siles [Cullen], 1799-III: 357).

Únicamente lo recogemos reformulado, durante este período, en textos que poseen una finalidad didáctica, cuando la resolución de un posible conflicto terminológico se hace prioritaria para el autor. El ejemplo (9) está tomado de Principios de cirugía, obra de carácter dialógico que publica Francisco Puig, cirujano del Real Colegio de Barcelona, para instrucción de los jóvenes médicos en la «verdadera cirugía» ${ }^{7}$.

(9) P. Qué es sangría? R. Es una operación hecha en los vasos sanguíneos con una lanceta a fin de sacar una porción de la massa de la sangre. Se distinguen dos especies de sangrías, una que se hace en las venas, y es la más común, nombrada: Phlebotomía; otra que se hace en las arterias, no muy ordinaria, y se llama: Arteriotomía (Puig, 1753: 142).

Esta obra de carácter didáctico presenta la doctrina como una verdad absoluta, puesta en boca del médico, pues es consciente de la importancia que reviste la aclaración del contenido conceptual que aportan los términos flebotomía y arteriotomía. Para ello emplea la reformulación parafrástica con los marcadores explicativos: nombrado y se llama, con los que Puig consigue la precisión semántica de ambos términos. Pero al mismo tiempo es interesante la información que da Puig acerca de la arteriotomía, al indicar que se trata de una práctica quirúrgica no demasiado frecuente. Gracias al marcador explicativo de carácter metalingüístico, el tejido discursivo de la reformulación contribuye eficazmente para poner en práctica la comprensión textual del contenido, proporcionándole mayor rigor significativo según se haga la incisión en una vena o en una arteria, mostrando en definitiva todo un alarde de precisión terminológica.

Así permanecerá la voz durante toda la etapa ilustrada y parte del siglo XIX, a veces acompañada de recursos textuales de la lengua común como las comparaciones que introducen en el discurso precisiones significativas:

(10) La sangría es un remedio conveniente y aun necesario quando hay frequencia o plenitud en el pulso, o algunas señales que indican que la sangre sube con más fuerza a los vasos de la cabeza. En estos casos algunos prácticos han preferido sangrías particulares como la arteriotomía, las escarificaciones en la nuca o la abertura de la yugular (Piñera y Siles [Cullen], 1799-III: 357).

Otras veces con reformulación no parafrástica o incluso sin reformular:

(11) La apoplejía debe atacarse por medio de la arteriotomia, de la flebotomia, de las sanguijuelas y de las ventosas. Después de esto, es menester des-

\footnotetext{
${ }^{7}$ Así lo afirma Pedro Virgili, director del Real Colegio de Cirugía de Cádiz cuando, dirigiéndose a Francisco Puig, escribe: «[...] con gran gusto he recibido la carta de Vmd. [...] por ver en ella desterrar de esse Hospital General la Cirugía de Vidos, que se ha observado hasta aquí, me alegraré que Vmd. continúe en ejecutar la verdadera Cirugía, siempre que los casos lo pidan, pero para esto es menester, que Vms. instruyan como Maestros a la Juventud, [...]», Aprobación de Pablo Barra, 1753: s/p, en Puig (1753). Este dato es revelador acerca de la importancia de la obra de Puig, así como de la novedad y renovación de la ciencia que introduce en su obra. Nótese que este médico catalán se refiere en (8) a la arteriotomía como una práctica que en los años centrales de la centuria es todavía mucho menos frecuente que la flebotomía.
} 
pertar la sensibilidad con eméticos, purgantes, lavativas irritantes, estimulantes, vejigatorios, sinapismos, movimientos, sacudimientos, etc. (Lorente [Capuron], 1831-II: 61)

A comienzos del XIX también recogemos algún empleo fluctuante como en (12) cuando este médico, traductor del Diccionario de Ciencias médicas de París, evita el empleo de la voz arteriotomía para referirse a la sangría de la arteria y la sustituye por «hemorragia arterial»:

(12) Curación de la fiebre intermitente atáxica. Aquí es donde se ve el triunfo de la terapéutica médica; y el cirujano que conserva los días de un herido, haciéndose dueño de una hemorragia arterial, no obra con más certeza y eficacia que el médico que cura por medio de la quina una fiebre intermitente perniciosa (Sánchez Núñez [Anónimo], 1819: 228). [Es traducción de un Diccionario de Ciencias médicas de París y un artículo sobre fiebres de Fournier y Vaidy, también traducido].

El concepto dieciochesco de la sangría practicada en una arteria pierde vigencia cuando la ciencia médica renuncia a la sangría como medio de curación. Es entonces cuando el significante arteriotomía desliza su significado para designar la actual voz de la cirugía: 'incisión quirúrgica de una arteria', totalmente desprovisto del concepto de sangría. Lo que nos lleva a afirmar que el término actual, presente hoy en los diccionarios especializados, es un neologismo de sentido.

\subsection{Poro}

El corpus también nos proporciona otras voces que, a pesar de haber permanecido desde antiguo en la lengua de la medicina, en un momento dado y debido a la impronta de algún cambio en el ámbito de especialidad, llegan a especializar su significado manteniendo el mismo significante que ya existía de antemano en el ámbito del conocimiento al que pertenecen. Es el caso del neologismo de sentido poro cuando a lo largo del siglo XVIII va evolucionando en su significado hasta especializarse con el significado que hoy tiene en el campo de la dermatología.

Es evidente que no se trata de una voz nueva en la literatura médica ${ }^{8}$ pues, aunque constatamos un empleo muy frecuente de la voz poro, para referirse a cualquiera de los poros que puede alojar el cuerpo humano o animal; sin embargo, es interesante observar que los ejemplos tomados en las últimas décadas del XVII para nombrar la voz dermatológica, presentan siempre el complemento del nombre: poro del cutis, como venía siendo habitual en los textos antiguos:

(13) $[\ldots]$ los quales encalvecen, y encanecen temprano, assí por la falta de humedad, como por tener los poros del cutis muy anchos, y manifiestos (Torre y Valcárcel, 1668 [1717]: 110).

(14) El medicamento resolutivo es de más tenue substancia, y más cálido que el anodino, por cuya razón penetra con facilidad dilatando los poros del cutis,

\footnotetext{
${ }^{8}$ La voz poro con el significado 'orificio por el que pasan fluidos' tiene larga tradición en nuestra lengua, el Fichero General de la Real Academia Española ofrece 389 cédulas, la primera datada en el Poema de Mio Cid. El DETEMA (Herrera: 1196) ya recoge poro con el significado de 'orificio cutáneo' en TRM s. XV.
} 
y atenuando los humores, los convierte en vapor, para que se evacuen por insensible transpiración (Robledo, 1687 [1703]: 64).

(15) Por el resolutorio al contrario, por los poros de la carne a los de la cutis, los quales son más densos, pero no se pueden aplicar los repelentes en todos los tumores al principio de la fluxión (Borbón, 1688 [1705]: 210).

Es quizá el carácter de precisión que supone la aclaración al referirse a la transpiración cutánea y el posible desconocimiento semántico que tal novedad implica, la que impulsa a los médicos dieciochescos a introducir en sus textos la forma simple poro mediante los rasgos de la reformulación:

(16) [...] los poros de el cutis, o papilas, como quiere Malpico, todos abiertos se exalan por sudor, de el mismo modo que cada día experimentamos con algunos liquores sutiles y espirituosos, que si los ponen en vasos, que no estén bien tapados, todos se exalan y no queda nada en el vaso (Juanini, 1679: XVI).

La revolución científica de los novatores llega a España en las décadas previas al comienzo de la nueva centuria; momento en que Juan Bautista Juanini ${ }^{9}$ escribe el texto de donde hemos extraído el ejemplo (16). Este médico italiano afincado en España y defensor de la iatroquímica, dota de sentido al nuevo concepto reformulándolo con estructuras discursivas que se encadenan sucesivamente buscando la precisión designativa. En primer lugar, y para lograr la recontextualización del término lo acompaña con el complemento del nombre poros del cutis de la misma manera que lo empleaban sus contemporáneos, como leemos en (13), (14) y (15); pero además refuerza el discurso reformulador con el sinónimo tradicional papilas, introducido con el marcador de equivalencia $o$; y por último, siguiendo el discurso clásico de la vulgarización científica, acerca hasta el lector el saber establecido mediante una cita de autoridad, que cierra el período al afirmar: «como quiere Malpico».

Con el avance del nuevo siglo observamos que poco a poco el complemento del nombre dejará paso a la forma simplificada poro de la medicina moderna, una vez que el término ha adquirido la precisión del significado ${ }^{10}$.

(17) El cutis tiene muchos agujeros manifiestos, como los de los ojos, oídos, nariz, boca, ano y partes pudendas; pero tiene otros innumerables que se llaman poros y solo se ven con el microscopio, [...] (Martínez, 1728: 61).

(18) [...] los vasos excretores sirven para que se haga sensible o insensiblemente la transpiración, para lo qual son de diferente magnitud y figura: cuyos orificios exteriores se llaman poros (Beaumont, 1728: 93).

Los dos ejemplos anteriores nos acercan textos coherentes con las nociones de contexto y registro, pues ambos explican el concepto y lo dotan de sentido, ofreciéndonos sendos espacios discursivos de interacción entre los interlocutores. Es evidente que el

\footnotetext{
${ }^{9}$ Este médico, muy destacado dentro de la corriente novatora, fue el precursor de la medicina social en España al adelantarla en sus obras: Discurso Político y Físico (1679) y Discurso Físico y Político (1689).

${ }^{10}$ El Diccionario de Domínguez de 1753 es el primer repertorio que recoge, en la segunda acepción de la voz poro, el mismo significado que nos proporciona el corpus: «2// Cada uno de los huecos imperceptibles que se hallan en la piel de los animales y por los cuales se efectúa la transpiración; $[\ldots] »$.
} 
Dr. Martínez, en el auge de la recepción en España de la nueva medicina, y ya casi superado el período novator, se ve obligado a salvar las diferencias léxico-semánticas que le plantea la lengua de especialidad cuando en su Anatomía completa del hombre, obra cumbre de la medicina novatora, se propone alcanzar la inmediatez comunicativa a fin de transmitir a sus lectores una perfecta comprensión del mensaje tal como nos lo muestra en (17). De igual manera procede Beaumont, médico de la real familia con Felipe V y Fernando VI, al emplear el marcador de reformulación explicativo en (18).

Estos ejemplos vienen a constatar un estilo discursivo propio de la etapa novatora, pues estos dos ilustres médicos, ambos al servicio de la corte en el primer tercio del siglo, abordan la construcción del discurso mediante la inclusión en la primera parte del enunciado, de un conjunto de rasgos definitorios que potencian los efectos persuasivos necesarios para captar la atención del lector, tales como la didacticidad, analogías, etc. Mientras que preservan la segunda parte del enunciado para introducir el neologismo objeto de la reformulación, explicitado en ambos casos con un verbo metalingüístico.

Gracias al generoso conjunto de textos que nos proporciona el corpus hemos podido hacer el seguimiento del neologismo de sentido poro a lo largo de todo el siglo. Entre los datos interesantes que espigamos en este acervo de textos médicos, llama nuestra atención que un autor como Beaumont, en una obra publicada una década más tarde que la del ejemplo (18), todavía reformula el término, aunque cambia el estilo al establecer un cierto distanciamiento entre el enunciado y su formulación, característico de la reformulación no parafrástica (19). Este nuevo empleo del discurso nos pone sobre la pista de lo que podríamos considerar un afianzamiento de la nueva voz en la literatura médica, donde habría ido introduciéndose y adquiriendo solidez semántica década tras década. Beaumont únicamente la reformula avanzando en la comprensión textual del contenido, con una aclaración de tipo descriptivo de la que se sirve para establecer la distancia semántica que hay entre la voz tradicional poro y la voz de la dermatología que aquí nos ocupa.

(19) y essa mecánica dispone todas las operaciones, de las quales la naturaleza necessita para sus usos, y essa explicación sigue passo a passo las experiencias, que la circulación nos enseña y nos da una idea justa de la disposición de los poros, que se encuentran a la superficie del cutis (Beaumont, 1739: 15).

Ya en la segunda mitad de la centuria, las traducciones de carácter divulgativo conceden importancia a la nueva voz de la dermatología. En ocasiones se trata de médicos consagrados, grandes teóricos de la medicina dieciochesca, como es el caso de Heister, autor de numerosas obras destinadas a un lector con rigor científico y verdadero especialista como médico ilustrado, que también publica alguna obra con fines divulgativos como expone en el prólogo de la obra de donde hemos tomado el ejemplo (20), es decir, en Fundamentos o Instituciones médicas breves y claras en latín y en español. Cuando reformula la voz con el verbo metalingüístico llamar, procurando la equivalencia conceptual de inclusión:

(20) $[\ldots]$ y por los pequeños e insensibles forámenes del cutis, que comunmente llaman poros, se transpira en grande cantidad, con gran provecho de nuestra sanidad (García Vázquez [Heister], 1751: 61). 
(21) Pequeñísimos forámenes que previenen salida a los pelos, transpiración y sudor: y dan entrada al mercurio, y a otras cosas, a quien el vulgo llama poros (García Vázquez [Heister], 1755: 53).

(22) [...] Stenón observó que a cada poro del cutis estaba baxo puesta su glándula, de la qual sale un vaso sudorífero, el que termina, en la superficie de la cutis (García Vázquez [Heister], 1755: 230).

Sin embargo, a medida que va avanzando la centuria constatamos la ausencia de reformulación en los textos que nos acercan las traducciones de los dos autores más exitosos de la medicina divulgativa dieciochesca: el franco-suizo Tissot y el inglés Buchan:

(23) Los poros de la cabeza tapados siempre con un emplasto grasiento y farináceo y algunas veces también los del rostro con pomadas cargadas con partículas dañosas, cuyos peligros ha dado muy bien a conocer Mr. Des Hais, son también causas que perjudican deteniendo la transpiración, la que impedida refluye a los órganos vecinos, y produce en ellos muchos y muy diversos males (Galisteo Felix [Tissot], 1786: 221).

En (23) el médico madrileño Felix Galisteo, traductor de Tissot, emplea la voz poro sin reformular, únicamente con la precisión anatómica al indicar poros de la cabeza o poros del rostro. Tampoco lo van a reformular ninguno de los traductores de la Medicina doméstica de Buchan:

(24) En este tiempo se padecen muchas enfermedades por defecto de transpiración, y pueden precaverse en algún modo por el correspondiente aumento de ropa, o vestirse de la que sea más proporcionada a promover la evacuación de los poros como algodón, o bayeta, \&c. (Alcedo [Buchan], 1785: 103).

(25) El estar en un quarto caloroso, el beber licores cálidos y el salir repentinamente al ayre frío mientras los poros están abiertos, es en mucho extremo peligroso (Sinnot [Buchan], 1785: 151).

Tampoco lo reformula Serrano Manzano cuando vierte al castellano la obra de Brown. El pasaje siguiente describe el proceso de contagio de la enfermedad con intervención de los poros cutáneos que ni el autor, ni el traductor reformulan; parece pues evidente que la voz de la dermatología estaba ya totalmente introducida en la literatura médica durante la etapa ilustrada en Europa:

(26) El contagio es una cierta materia imperceptible de una naturaleza desconocida, como lo son los más de los fenómenos de la naturaleza, y únicamente susceptible de indagarse en algún modo en sus evidentes efectos. Tomada del cuerpo de una persona inficionada, o de algún otro cuerpo tosco (tal como los vestidos u otras cosas semejantes en donde casualmente puede estar anidada), y recibida dentro de un cuerpo sano fermenta sin mutación alguna en los sólidos y en los fluidos, llena todos los vasos, y después gradualmente es arrojada por los poros de la superficie (Serrano Manzano [Brown], 1800-II: 23).

Sin embargo, no ocurre lo mismo en las traducciones que por los mismos años se hacen de la obra de Brown en la Nueva España, cuando el traductor evita en todo momento el empleo del término poro bien reformulándolo o bien empleando la voz cutis mediante 
diversos sintagmas aclaratorios, algunos muy empleados en España desde comienzos del siglo ilustrado como vasos del cutis, vasos cutáneos ${ }^{11}$, vasillos de la superficie, etc.:

(27) Porque reteniendo la atonía y relaxación de los vasillos de la superficie la transpiración, acumulan el calórico baxo la cutis (Anónimo [Brown] Méx., 1802: 58).

(28) Nace el temblor que distingue el acceso de las enfermedades esténicas de la transpiración disminuida en los vasos cutáneos por una diátesis (Anónimo [Brown] Méx., 1802: 48).

(29) El contagio recibido en el cuerpo y difundido por todo él, es retenido con la transpiración en los vasos de la cutis (Anónimo [Brown] Méx., 1802: 49).

(30) Los vasos perspiratorios de la cutis (Anónimo [Brown] Méx., 1802: 52).

(31) La grande excitación de las fibras que forman el texido de los vasillos de la superficie del cuerpo y la densidad de la cutis, causan aquella sequedad que produce el temblor y la sensación de frío.

La pervivencia, de casi un siglo, de estas palabras en los textos de la dermatología nos lleva a pensar con Sophie Moirand (2000: 2689) que los períodos de acusada divulgación pueden llegar a desarrollar una «mémoire interdiscursive» de tal manera que algunas palabras, algunos semas, algunas construcciones sintácticas se afianzan en lugares privilegiados del texto, dando lugar a un cierto tipo de subgéneros discursivos.

\section{CONCLUSIÓN}

Los textos que nos proporciona el corpus compilado nos han permitido delimitar algunas voces neológicas de la medicina del siglo XVIII insertas en ricos períodos discursivos propios de la reformulación, mayoritariamente parafrástica, aunque también hemos podido espigar algunos ejemplos de reformulación no parafrástica. Estas nuevas voces se presentan en los textos como elementos imprescindibles para conseguir el objetivo prioritario de la transmisión científica, esto es, una transmisión plenamente fiable.

Para rastrear los procedimientos de la reformulación hemos propuesto dos sincronías que tienen importancia capital para el desarrollo de la nueva ciencia en el siglo ilustrado, además estos dos momentos nos han permitido detectar la evolución de las marcas de la reformulación en el siglo XVIII.

\section{REFERENCIAS BIBLIOGRÁFICAS}

BACH, Carme (2005): «Los marcadores de reformulación como localizadores de zonas discursivas relevantes en el discurso especializado». Debate terminológico, 1 [Fecha de consulta: 10/06/2019: http://hdl.handle.net/10230/6164].

BRUMME, Jenny (2001): La historia de los lenguajes iberorrománicos de especialidad la divulgación de la ciencia: actas del II Coloquio Internacional, 27-29 de mayo de

\footnotetext{
${ }^{11}$ Estas voces las emplea regularmente Manuel de Porras en su obra Anatomía galénico-moderna, de 1916.
} 
1999 "La historia de los lenguajes iberorrománicos de especialidad”, Barcelona, Iberoamericana.

CAlsamiglia, Helena (2000): «Decir la ciencia las prácticas divulgativas en el punto de mira», Discurso y Sociedad, Barcelona, Gedisa, 2, pp. 2-18.

Herrera, María Teresa, dir. (1996): Diccionario español de textos médicos antiguos, Madrid, Arco/Libros [DETEMA].

Cortés Gabaudan, Francisco y Jesús Ureña Bracero (2011): Diccionario médicobiológico, histórico y etimológico [DICCIOMED] [Fecha de consulta 15/10/2019: https://dicciomed.usal.es].

DomíngueZ, Ramón Joaquín (18535): Diccionario Nacional o Gran Diccionario Clásico de la Lengua Española (1846-47), Madrid-París, Establecimiento de Mellado, 2 vols.

Real Academia Nacional de Medicina (2012): Diccionario de términos médicos, Madrid, Panamericana.

EBERENZ, Rolf (1991): «Castellano antiguo y español moderno», Revista de Filología Española, LXXI, pp. 92-106.

Fuentes, Catalina (1994): «Usos discursivos y orientación argumentativa: de hecho, en efecto, efectivamente», Español Actual, 62, pp. 5-18.

GÓMEZ DE ENTERRÍA, Josefa (2013): «Higiene y salud en las traducciones médicas del francés al español durante el siglo XVIII»,Panace@, 14, 38, pp. 287-295.

GÓMEZ DE ENTERRÍA, Josefa (en prensa): El vocabulario de la medicina en el siglo XVIII, Berna, Peter Lang.

MARTín Zorraquino, María Antonia y José Portolés LÁZARO (1999): «Los marcadores del discurso», en Ignacio Bosque y Violeta Demonte, dirs., Gramática descriptiva de la lengua española, Madrid, Espasa, 3, pp. 4041-4214.

MoIRAND, Sophie (2000): «Les manifestations discursives dialogiques de la rencontre entre sciences, médias et politique», en José Jesús Bustos Tovar, coord., I Simposio internacional de análisis del discurso, Madrid, Visor, 2, pp. 2681-2698.

OESTERREICHER, Wulf (2004): «Textos entre inmediatez y distancia comunicativas. El problema de lo hablado escrito en el siglo de oro», en Rafael Cano, coord.., Historia de la lengua española, Barcelona, Ariel, pp. 729-760.

REAL ACADEMIA ESPAÑOLA: Fichero General de la Lengua española [Fecha de consulta 15/10/2019: http://web.frl.es/fichero.html].

ROSSARI, Corinne (1994): Les opérations de reformulation, Berna, Peter Lang.

Roulet, E. (1987): «Complétude interactive et connecteurs reformulatifs», Cahiers de Linguistique Française, 8, pp. 111-139.

SÁNCHEZ NúÑEZ, Lorenzo (1819): Diccionario de fiebres esenciales / compuesto y traducido del artículo de fiebres y otros varios contenidos en el diccionario de ciencias médicas actualmente está formando en que una sociedad de sabios por D. Lorenzo Sánchez y Núñez, Madrid, Repullés.

TERREROS Y PANDO, Esteban de (1767) [1786, 1787, 1788]: Diccionario castellano con las voces de ciencias y artes y sus correspondientes en las tres lenguas francesa, latina e italiana [...], Madrid, Viuda de Ibarra, 3 vols.

\section{CORPUS TEXTUAL}

AlCEDO, Antonio de [BuChan, William] (1785): Medicina doméstica o Tratado completo del método de precaver y curar las enfermedades con el régimen y medicinas simples: y un apéndice que contiene la famacopea [...] escrito en inglés por Jorge 
Buchan; traducido en castellano por [...] D. Antonio de Alcedo, Madrid, Imprenta de D. Antonio Sancha [BDH].

Anónimo [Brown, J.] (1802): Epitome de los elementos de medicina del Dr. Juan Brown. [Traducido por J. R. en México]. Puebla, s. 1.

BEAUMONT, Blas (1728): Exercitaciones anatómicas y essenciales operaciones de cirugía: con un breve resumen de los instrumentos y vendages, el modo de circular la sangre, un riñón extraordinario y algunos remedios: con las láminas finas y más essenciales de la Anatomía e instrumentos de las operaciones, Madrid, Imprenta del Convento de Nuestra Señora de la Merced.

BEAUMONT, Blas (1739): El bien del hombre buscado, y hallado en el mismo: con las reflexiones de anathomía, y cirugía, casos de práctica muy particulares sobre las operaciones, enfermedades. Tomo II de su Anathomía, Madrid, Imprenta de Joachín Sánchez y a costa de Bartolomé Torrano.

Belmonte y Segura, Miguel Francisco (1730): Examen phísico, chyrúrgico, médico, moderno para jóvenes chyrúrgicos y médicos: en el qual se hallarán los más cuerpos sublunares delineados, y definidos chýmicamente [...] según la secta Tacheniana [...] y finalmente se explica cómo enferman chyrúrgica, y médicamente los humanos vivientes [...] su compositor, y autor don Miguel Francisco Belmonte y Segura, Granada, Imprenta de Joseph de la Puerta.

BORBÓN, Felipe (1705): Medicina y cirugía doméstica, necessaria a los pobres y familiar a los ricos: trascripta del Medico caritativo, con algunos remedios de otros autores: con escolios en las materias y afectos que se tratan, assí chirúrgicos, como médicos por el licenciado Felipe Borbón, añadido en esta última impressión las Flores de Guido, Valencia, Jayme de Bordazar y Artazú.

CABRIADA, J. (1687): De los tiempos y experiencias el mejor remedio al mal por la novaantigua medicina: carta philosophica medica chymica, Madrid, Oficina de Lucas Antonio de Bedmar y Baldivia.

Galisteo Xiorro, Félix [TISSOT, Samuel Auguste André David] (1786): Aviso a los literatos y poderosos acerca de su salud, o Tratados de las enfermedades más comunes a esta clase de personas con varias observaciones sobre el cólico plúmbeo o metálico, el vómito negro y otros diferentes objetos de Medicina por Mr. Tissot [...] obra traducida del francés por D. Félix Galisteo y Xiorro. Madrid, Imprenta de Benito Cano.

GARCÍA VÁzQUEZ, Andrés [HEISTER, Lorenz] (1751): Fundamentos o Instituticiones médicas en latín, y en español, compuestas por el doctor D. Lorenzo Heister [...] y las publica D. Andrés García Vázquez, Madrid, Juan de Zúñiga.

GARCÍA VÁZQUEZ, Andrés [HEISTER, Lorenz] (1755): Compendio anatómico que brevíssimamente comprehende y explica esta ciencia por don Laurencio Heister. Traducido de la lengua latina por Don Andrés García Vázquez, Madrid, Herederos de don Francisco Rodríguez.

JUANINI, Juan Bautista (1679): Discurso político y phísico, que muestra los mouimientos, y efectos, que produce la fermentación, y materias nitrosas en los cuerpos sublunares y las causas que perturban las saludables, y benignas influencias, que goza el ambiente de esta imperial villa de Madrid, corte de nuestro cathólico monarca Carlos II que Dios guarde, Madrid, Antonio Goncález [sic] de Reyes.

LE PREUX, Ricardo (1717): Doctrina moderna para los sangradores: en la qual se trata de la flebotomía, y ateriotomía, de la aplicación de las ventosas, y de las enfermedades de la dentadura, Madrid, Imprenta de Francisco del Yerro. 
LORENTE, Higinio Antonio [CAPURON, J.] (1831): Nuevos elementos de medicina: arreglados a la nosografía filosófica y dispuestos para el uso de los alumnos, Barcelona, Imprenta de J. Mayol y C. a, 2 vols.

MARTÍNEZ, Martín (1732 [1797]): Examen nuevo de cirugía moderna: nuevamente enmendada y añadido con las operaciones chyrúrgicas. Madrid, Imprenta de la Viuda e hijo de Marín.

MARTíneZ, M. (1728 [1775]) Anatomía completa del hombre con todos los hallazgos, nuevas doctrinas y observaciones raras hasta el tiempo presente y muchas observaciones necesarias para la cirugía, Madrid, Miguel Escribano.

MASSONEAU, Juan (1722): Cirugía natural, dada a luz por el supremo autor en la creación del hombre: dirigida por la circulación de la sangre, Madrid, Imprenta de Juan de Ariztia.

MonRAVÁ Y RoCA, Antonio (1725-1728): Breve curso de nueva cirugía [...] por don Antonio de Monravá y Roca [...] fundador de la Nueva Academia Phýsico Cirúrgica [...], leido en dicha Academia con nuevas doctrinas, derivadas de nuevos principios phýsicos [...]; en dos tomos dividido y en forma de dialogo escrito, primero tomo [II tomo], Lisboa Occidental, Imprenta Música: véndese en casa de su autor, en la rúa dos Escuderos, 2 vols.

PIÑERA Y SiLES, Bartolomé [CULLEN, William] (1799): Elementos de medicina práctica del doctor Guillermo Cullen [...] traducidos de la quarta y última edición inglesa al francés, con notas [...] por Mr. Bosquillon [...] y del francés al castellano, añadiéndoles varias notas por D. Bartolomé Piñera y Siles, Madrid, Imprenta de Don Benito Cano, 1788-1791, 4 vols. [Nota: este es el tomo III reeditado y aumentado en 1799].

Porras, M. (1716 [1733]) Anatomía galénico-moderna, Madrid, Pedro Joseph Alonso y Padilla.

PUIG, Francisco (1753): Principios de cirugía, con los quales se instruyen los jóvenes, que se destinan a la professión de esta grande arte, en el Real Hospital General de Barcelona compuestos por Francisco Puig, Barcelona, Imprenta de Teresa Piferrer Viuda.

RoBledo, Diego Antonio de (1687 [1703]): Compendio cirúrgico útil y provechoso, Barcelona, Imprenta de Rafael Figueró.

SERrano MANZANO, Joaquín [BROWN, J. y Du MARSAIS] (1800): Elementos de medicina de doctor Juan Brown, trad. del latín al inglés por el autor y del inglés al castellano por el doctor don Joaquín Serrano Manzano. Contiene también la Lógica de Du Marsais, Madrid, Imprenta Real, 2 vols.

Sinnot, Pedro [Buchan, William] (1785-1786): Medicina doméstica o Tratado completo sobre los medios de conservar la salud, precaver y curar las enfermedades por Guillelmo Buchan [...] traducido del inglés al castellano por don Pedro Sinnot [...] adicionada con algunas de las notas (también traducidas al castellano) que puso Mr. Duplanil, Madrid, Imprenta de Andrés Ramírez, 5 vols.

TORRE Y VALCÁRCEL, Juan de la (1715): Espejo de la philosophía y compendio de toda la medicina, theórica y práctica por el doctor don Juan de la Torre [...]. Añadido y enmendado en esta tercera impressión el Tratado de morbo gálico, con un antidotario de pózimas, bevidas, xaraves, píldoras, vnguentos, mercurio zarza, palo santo y estufa, pertenecientes a su curación, Pamplona, Francisco Picart. 